\title{
BREAKS AND LONG TERM TRENDS OF TIDAL CHARACTERISTICS IN THE SOUTHERN GERMAN BIGHT
}

\author{
Sabine Jenning ${ }^{1}$, Hartmut Hein ${ }^{2}$, Stephan $\mathrm{Mai}^{3}$ and Holger Schüttrumpf ${ }^{4}$
}

\begin{abstract}
The recent influence of climate change and land subsidence on the water levels and tidal characteristics in the German Bight is documented by regional tide gauge observations. However, in any long time series the chance arises that measurement conditions may change over time. Some of these changes occur instantaneously like the change of the sensor or corrections of the zero-point of the gauge. Other changes occur subtly, such as alterations of the regional morphology and therefore the mode of behavior at the measurement site. We present long-term changes of tidal characteristics in the waterways of the southern German Bight, by detecting abrupt breakpoints and resulting homogenized trends. In order to understand processes of the changes of the tidal characteristics significant trends in the time series for measured and calculated tidal parameters are analyzed.
\end{abstract}

Keywords: long term trends; breakpoints; tidal characteristics

\section{INTRODUCTION}

The recent influence of climate change and land subsidence on the water levels and tidal characteristics in the German Bight is documented by regional tide gauge observations (Wahl et al. 2011, Hein et al. 2011a, Hein et al. 2011b). Figure 1 shows the rates of the sea level rise for the whole world (solid line) and for the German Bight (dashed line) as an example for a local observation area. The difference between the two is evident, partially they behave even contrary. For the deduction of adaptation strategies it is important to analyze local areas separately.

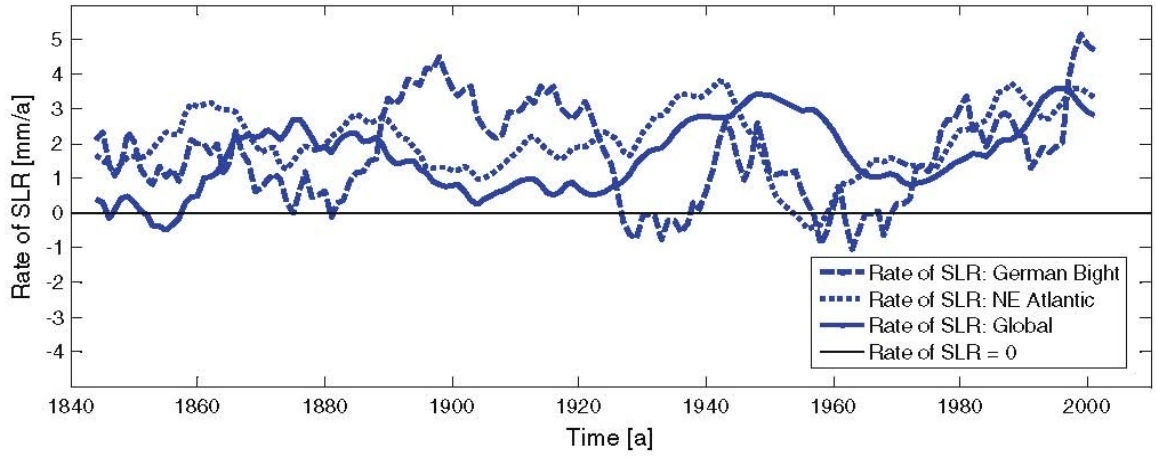

Figure 1. Rates of the sea level rise (SLR) global (solid line) and for the German Bight (dashed line) (from Wahl et al. 2011)

However, in any long time series as well as in series of tide gauge measurements the chance arises that measurement conditions may change over time. Some of these changes occur instantaneously, for example the change of the sensor or corrections of the zero-point of the gauge. On the other hand other changes occur subtly, such as alterations of the regional morphology and therefore the mode of behavior at the measurement site.

We present long-term changes of tidal characteristics in the waterways of the southern German Bight, by detecting abrupt breakpoints and resulting homogenized trends. The aim of the present analysis is to show if there are significant trends in the time series for measured and calculated tidal parameters in order to understand processes of the changes of the tidal characteristics. This description of the tidal characteristic by five parameters exceeds the pure consideration of the mean sea level (MSL). In a tide-dominated region, like the German Bight, each parameter has the potential to behave differently than the MSL.

\footnotetext{
${ }^{1}$ Institute for Hydraulic Engineering and Water Resources Management, RWTH Aachen University, Mies-van-derRohe-Straße 1, 52056 Aachen, Germany, jenning@iww.rwth-aachen.de

${ }^{2}$ Federal Institute of Hydrology, Am Mainzer Tor 1,56068 Koblenz, Germany, hein@bafg.de

${ }^{3}$ Federal Institute of Hydrology, Am Mainzer Tor 1, 56068 Koblenz, Germany, mai@bafg.de

${ }^{4}$ Institute for Hydraulic Engineering and Water Resources Management, RWTH Aachen University, Mies-van-derRohe-Straße 1, 52056 Aachen, Germany, schuettrumpf@iww.rwth-aachen.de
} 


\section{DATA AND TECHNIQUES}

\section{Data for the analysis}

For the present analysis the long-term datasets of 8 tide gauges in the southern German Bight are used, providing information about the value and the time of occurrence of the high-water and the lowwater level. The measured data come from the archives of the Waterways and Shipping Administration of the Federal Government (German: WSV). These records provide an indispensable base for the todays and the future research on climate impact. The dataset was newly quality-controlled by a recalculation of geodetic information about the zero-point of the sensors (Sudau and Weiss 2010). Hein (2010) showed the improvement in the accuracy of the dataset.

Based on this dataset of tidal high water levels $(T h w)$ and low water levels (Tnw) other specific parameters of the tide were calculated (see Figure 2). The results are time series of the tidal ranges (Thb), the flood periods $\left(T_{F}\right)$ and the ebb periods $\left(T_{E}\right)$, so that we can describe the main components of the tidal characteristics by these five parameters.

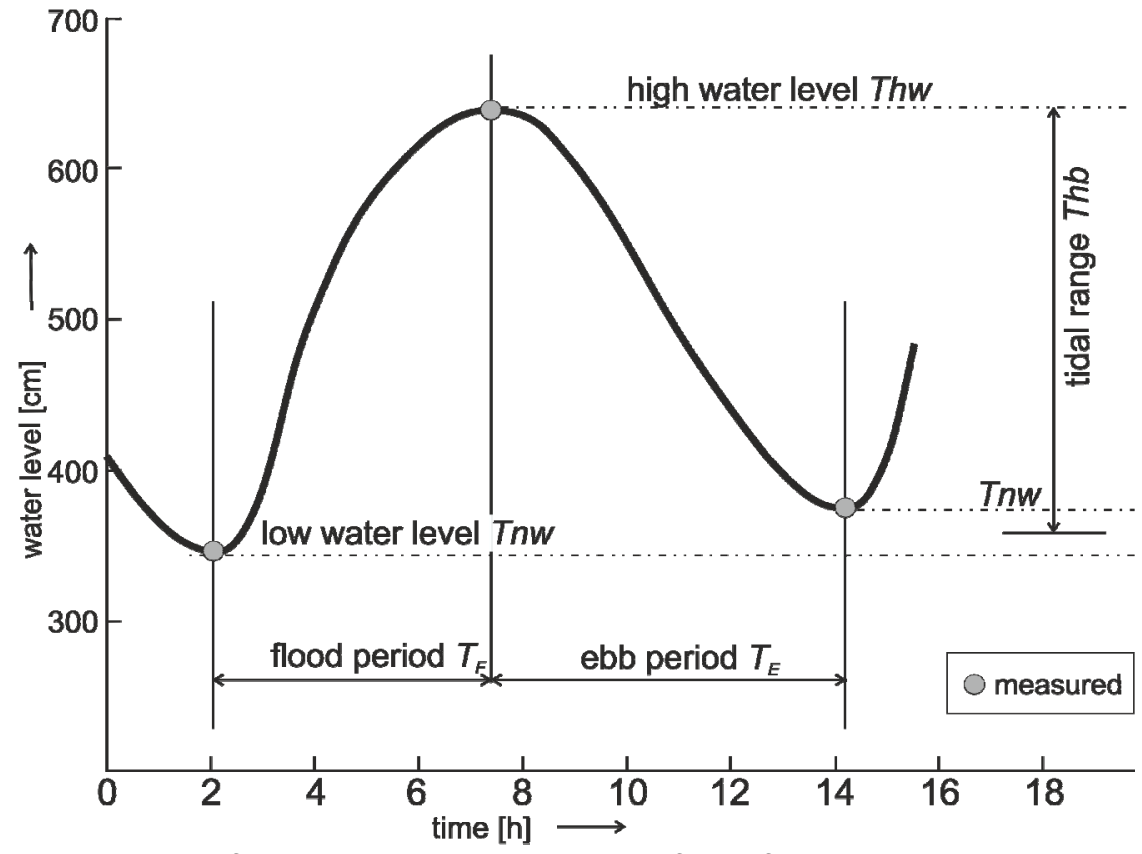

Figure 2. Schematic image of the tide and tidal parameters (defined after DIN 4049-3 (1992))

\section{Techniques for the breakpoint analysis}

By the use of three different algorithms the applicability of breakpoint analysis on tidal characteristics in the coastal region is tested. Therefore, breakpoints were calculated both for the values itself and for the stochastic variability. These three tested algorithms are:

- the standard normal homogeneity test (SNHT) by Alexandersson (1986),

- a nonparametric variant of the SNHT using the Wilcoxon ranksum statistics of the values (NPW) by Reeves et al. (2006) and

- $\quad$ an innovative approach based on fuzzy logic.

Within the first two named algorithms a value for a testing statistics is calculated for different positions $c$ using a time series with the length $n$. The algorithms detect a possible change point, if the maxima of the testing statistics exceed a critical value which is depending on the sample size and a selectable significance level. In Figure 3 the graphical interpretation of these calculations and the following explanations is given. 


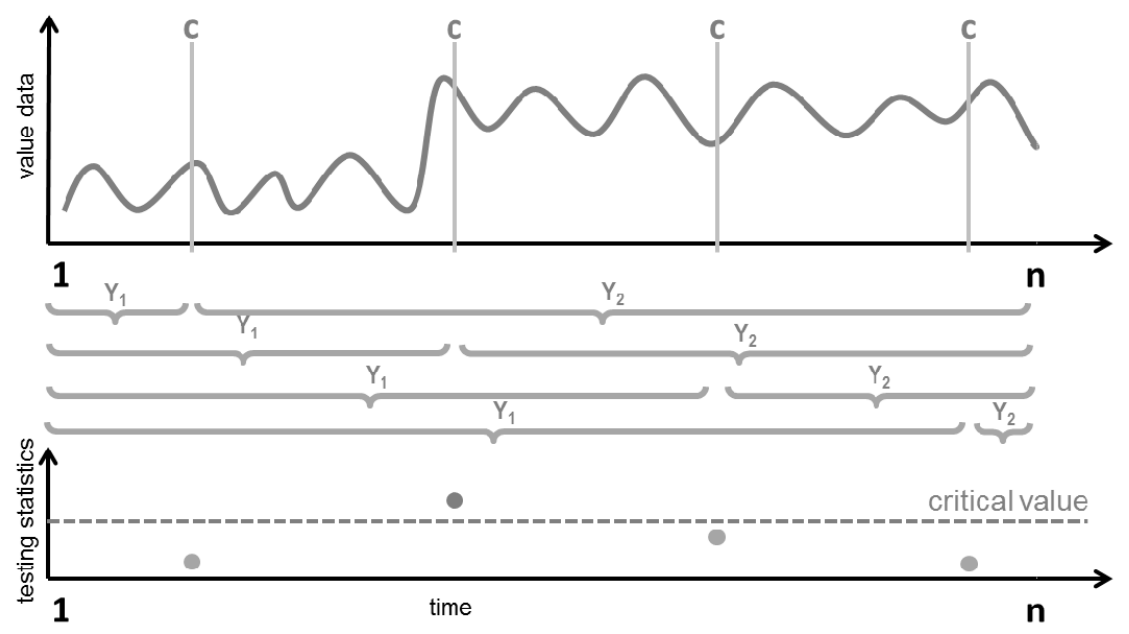

Figure 3. Schematic image using the algorithms SNHT and NPW for break point analysis

The first method for the breakpoint analysis is the standard normal homogeneity test (SNHT) by Alexandersson (1986) whereby the present modification comes from Reeves et al. (2006). With equation (1) the modified standard deviation $s_{p}$ is calculated using the standard deviation before $\left(s_{l}\right)$ and after $\left(s_{2}\right)$ the position of $c$. This is used in equation (2) for the calculation of the testing statistic $T_{c}$ where $\bar{Y}_{1}$ represent the mean before and $\overline{Y_{2}}$ the mean after the possible breakpoint at the position $c$. Equation (3) shows the hypothesis for the statistical test. If the squared maximum of the testing statistic $T_{c}$ exceeds the critical value $T_{\max }^{2}$ a breakpoint is detected.

$$
\begin{gathered}
s_{p}=\left[\frac{(c-1) s_{1}^{2}+(n-c-1) s_{2}^{2}}{n-2}\right]^{1 / 2} \\
T_{c}=\frac{\overline{Y_{1}}-\bar{Y}_{2}}{s_{p} \sqrt{c^{-1}+(n-c)^{-1}}} \\
T_{\max }^{2}=\max _{1 \leq c<n} T_{c}^{2}
\end{gathered}
$$

The second tested algorithm is a non-parametric variant of the standard normal homogeneity test NPW by Reeves et al. (2006). Within this variant not the measured data is used for the analysis but their ranks $r_{t}$. Equations (4) and (5) show the calculation of the testing statistic $W_{c}$ and the hypothesis for the comparison with the critical value $W_{\max }$.

$$
\begin{gathered}
W_{c}=12 \frac{\left[\sum_{t=1}^{c} r_{t}-c(n+1) / 2\right]^{2}}{c(n-c)(n+1)} \\
W_{\max }=\max _{1 \leq c<n} W_{c}
\end{gathered}
$$

The third method for the breakpoint analysis is an innovative approach based on fuzzy logic. It was developed within the project and will be refined during further investigations. The approach with fuzzy logic uses the principal of pattern detection within a time series. That means that one certain event can occur again after a certain pattern. In this special case the fuzzy system is trained with the properties (time series) of two gauges and the time series of a third gauge is predicted. Because the real 
measured data on the predicted gauge is known the comparison between predicted and measured values is possible. Significant differences between them might give the hint for a breakpoint. Two different methods were tested. The first proceeding was to use an increasing training period and a decreasing prediction period. Here the problem was that at the beginning of the calculations the prediction period was oversized in comparison with the small training period. On the other hand at the end of the calculations the training period was oversized in comparison with the small prediction period. Also the computation time increased. So the second proceeding is to use a fixed moving window. That means that the training and the prediction period have the same size all the time. The fuzzy system forgets things that are not in the training period any more. An advantage is that the computation time decreased.

\section{SELECTED RESULTS AND DISCUSSION}

\section{Results breakpoint analysis}

Our results show both: breakpoints in the tidal characteristics at individual measurement sites, as well as one breakpoint in the overall region. The first observation reflects that the waterways are strongly modified by local anthropogenic acting. The first two algorithms are tested with the annual means of the high and low water levels of the gauge Borkum Südstrand in the west of the German Bight. For the Fuzzy Logic approach the daily measured data is used, that means approximately two high and two low tides occur per day. Example results will be given for the three gauges Borkum Südstrand, Emden Neue Seeschleuse and Bremerhaven (see Figure 4).

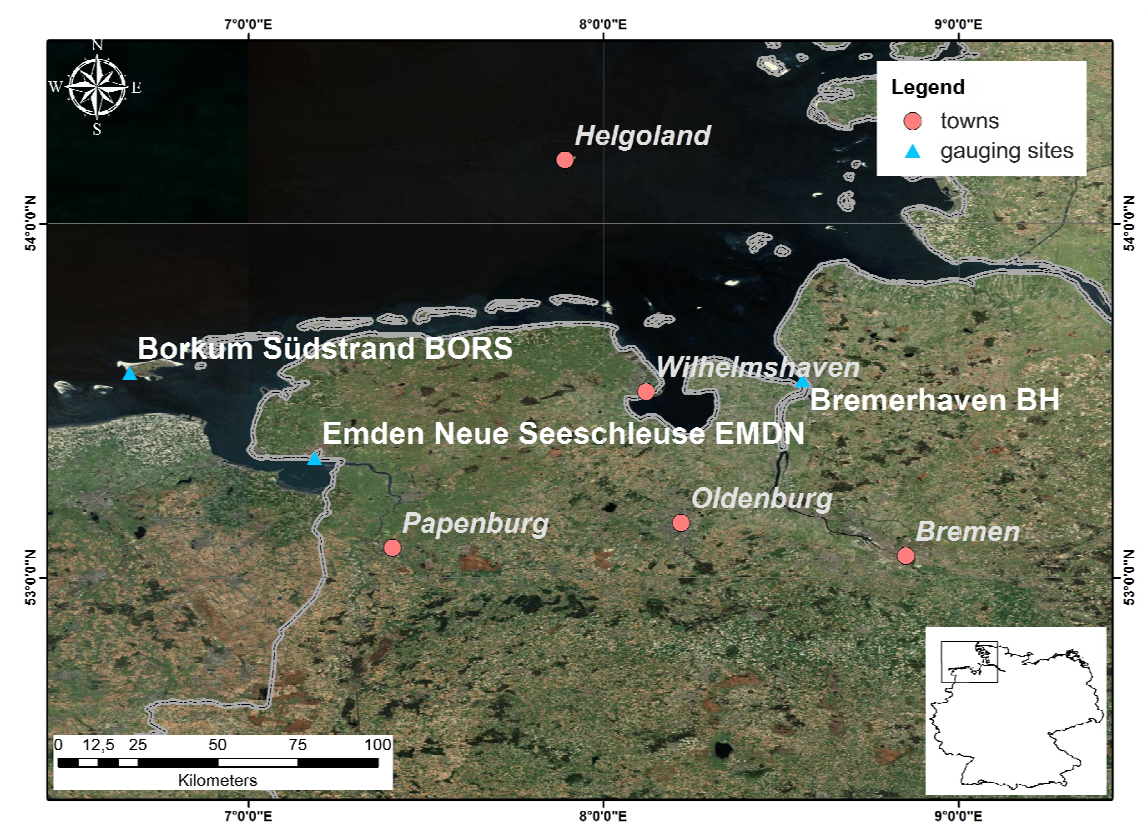

Figure 4. German Bight and location of the gauges Borkum Südstrand (BORS), Emden Neue Seeschleuse (EMDN) and Bremerhaven (BH)

The following Figure 5 shows the results for the first two statistical methods SNHT (b) and NPW (c) for the breakpoint analysis. Input data are the annual means of the high (solid blue) and low (dashed green) water level from 1935 till 2010 (see Figure 5(a)). 
Borkum Südstrand: annual mean Thw and Tnw (a), result SNHT (b) and result NPW (c)
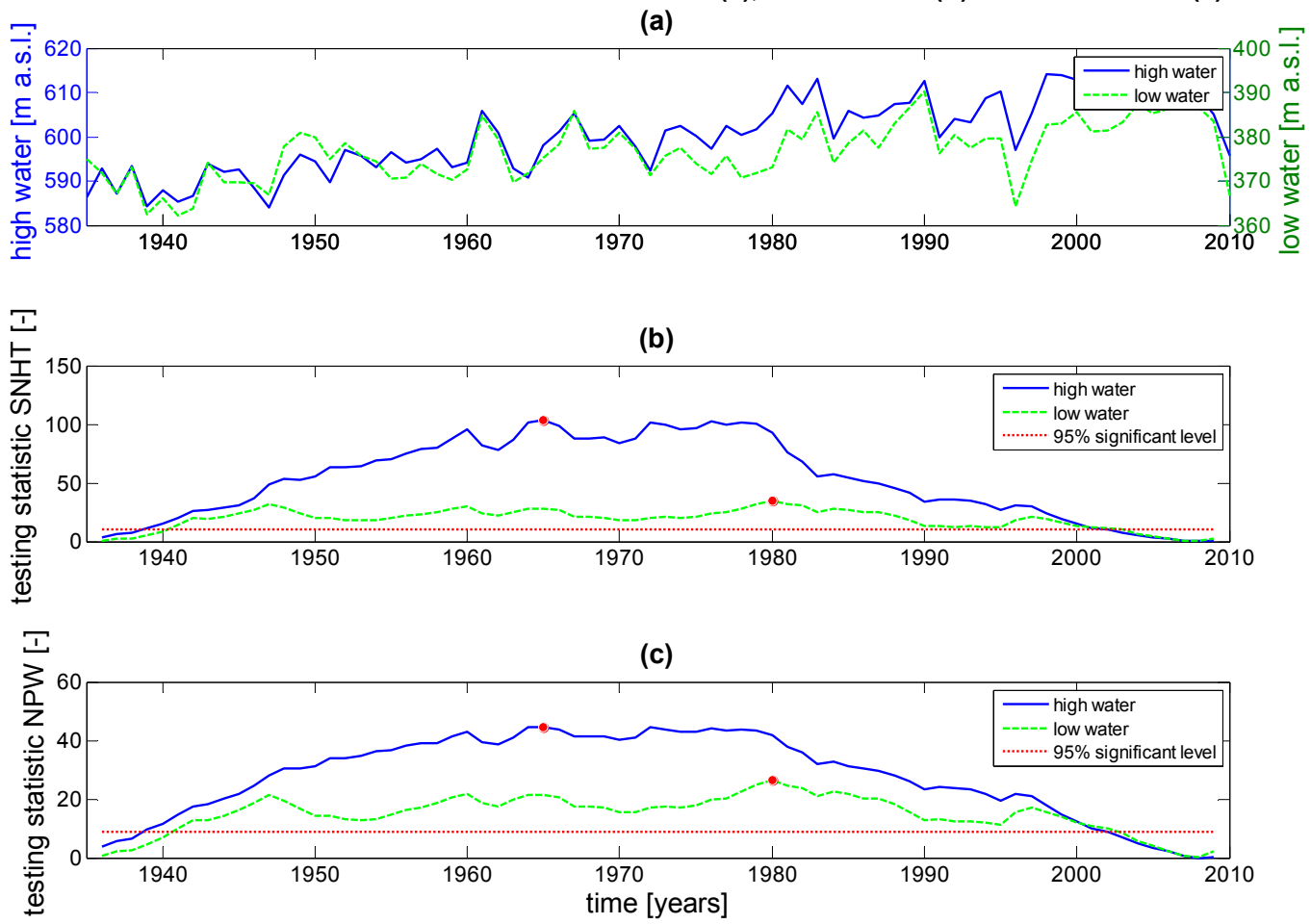

Figure 5. Gauge Borkum Südstrand, 1935 - 2010; (a) annual mean high (solid blue) and low water level (dashed green); (b) result testing statistic SNHT; (c) result testing statistic NPW

The maxima of the testing statistics are indicated with the red points. For both tests the peaks occur in the year 1965 for the high water level and in 1980 for the low water level. All detected maxima exceed the $95 \%$ significance level (dashed red line). That means statistically breakpoints are detected. The next step within the data preparation is the plausibility check to find out what might be the reason for the changes. In the register of the gauge and its history nothing is remarked for these years. Other reasons have to be found under consideration that the data is already quality controlled as mentioned before. Beside this geodetic quality control other changes within the data management might lead to the changes in the time series. Especially abrupt changes can be detected with these two algorithms.

However, also inherent physical changes may cause breakpoints. One indication of such break is the second peak of the testing statistics in the years 1979/80. This maximum occurs in several time series of different gauges all over the observation area, also at Borkum Südstrand. This second behavior must be interpreted as the result of a mode change of the tidal characteristics of the North Sea. This mode changes is comparable with known phenomena in the North Atlantic region. This breakpoint is also reflected in investigations of the ecosystem of the Wadden Sea (Eriksson et al., 2010). However, the breakpoint analysis allows detecting this breakpoint in tidal characteristic for the first time.

The Figure 6 and Figure 7 below show the results for the fuzzy logic approach for the two proceedings. In both cases two time series are used to train the fuzzy logic system. The time series for the third gauge are predicted for the given time vector. That means the results for the gauge Emden Neue Seeschleuse (solid blue) are predicted from the training with the gauges Borkum Südstrand (dashed green) and Bremerhaven (dotted red). The other results emerge accordingly. The values on the ordinate are the differences between the calculated and the measured data.

The training period for the results in Figure 6 increases by one month with each time step within the algorithm. In the beginning of the predicted time series the differences are high for BORS and BH due to the short training period and the long prediction period. The more the system knows by the means of the range of the observed values, the smaller the differences become. The newly increasing at the gauge Borkum Südstrand from 2005 till the end indicates a quality problem at the measurement site. As for the statistical techniques the next step is the plausibility check to validate whether these peaks can give a hint on breaks within the time series. 
Breakpoint analysis using Fuzzy Logic for Thw (a) und Tnw (b) gauges BH, BORS and EMDN, increasing training period

(a) high water

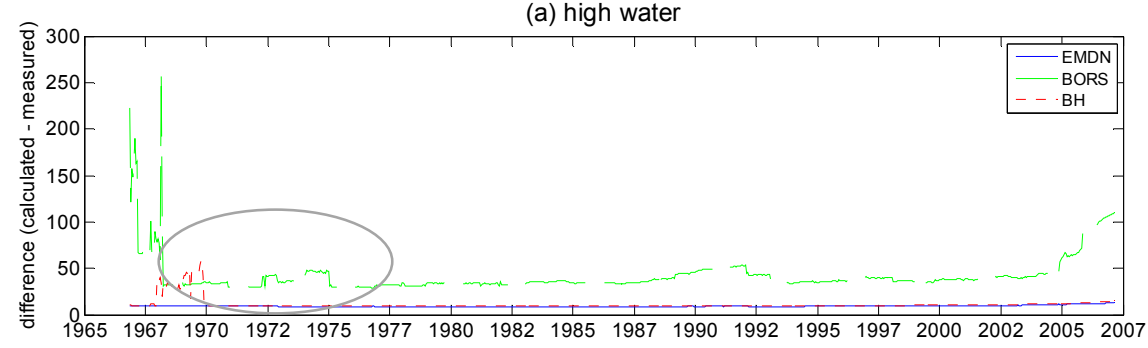

(b) low water

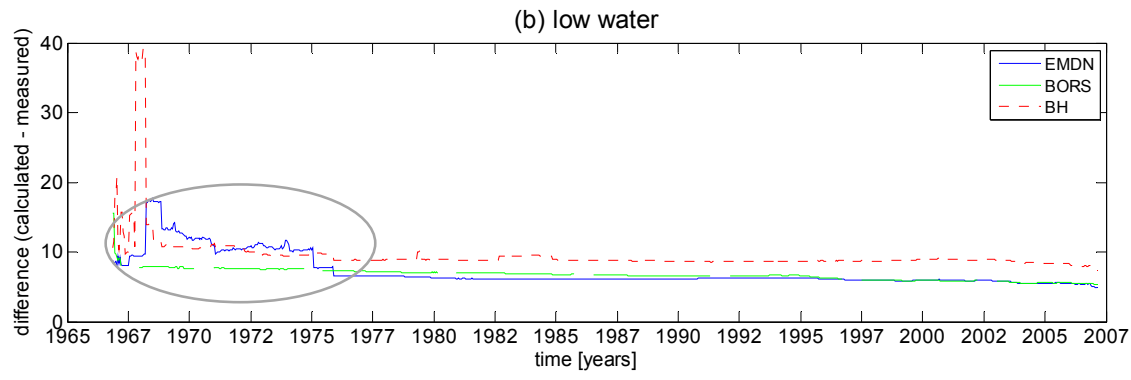

Figure 6. Results fuzzy logic for gauges Borkum Südstrand (BORS), Emden Neue Seeschleuse (EMDN) and Bremerhaven $(\mathrm{BH})$ using increasing training period

In the second proceeding (Figure 7) the training and prediction period are shifted by one month with each time step. This means that only one window in the whole time series is used for the analysis. The denotations of the resulting time series with the training and the prediction is the same as in the first proceeding. The values on the ordinate are also the differences between the calculated and the measured data. The results show peaks in the differences over the whole time series. Due to the shifting of the training and the prediction period the fuzzy system 'forgets' events, that are no longer in the training period.

Breakpoint analysis using Fuzzy Logic for Thw (a) und Tnw (b) gauges BH, BORS and EMDN, fixed moving window

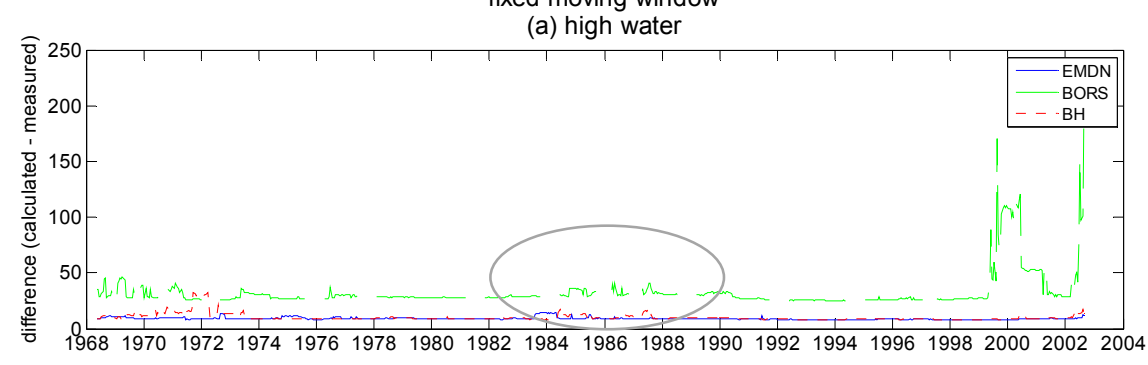

(b) low water

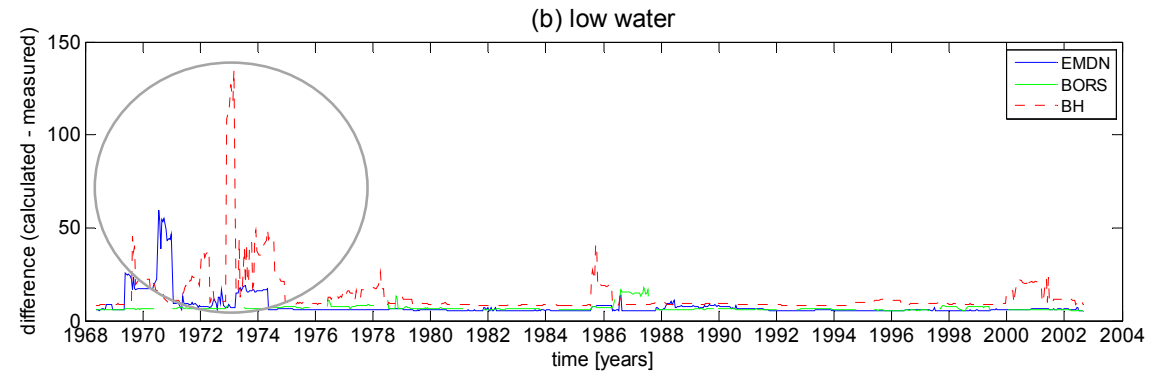

Figure 7. Results fuzzy logic for gauges Borkum Südstrand (BORS), Emden Neue Seeschleuse (EMDN) and Bremerhaven (BH) using fixed moving window 
The next step within this analysis is also the plausibility check to verify if these peaks can detect breakpoints in the time series of the daily measured data. The newly increasing at the gauge Borkum Südstrand at the end of the time series is even more distinctive. This again might give a hint on a quality problem at the measurement site.

\section{Results trend analysis}

Long term trends in tidal characteristics become significantly different if the detected breakpoints are taken into account. In Figure 8 are again the annual means of the high water level (a) and the low water level (b) from the gauge Borkum Südstrand from 1935 till 2010.The dashed line represents the linear trend for the whole time series. Within the two statistical tests SNHT and NPW two breakpoints were detected: 1965 in the time series of the high water level and in 1980 for the low water level. Separated at these times, the solid lines represent the linear trends for the two time series.

(a)

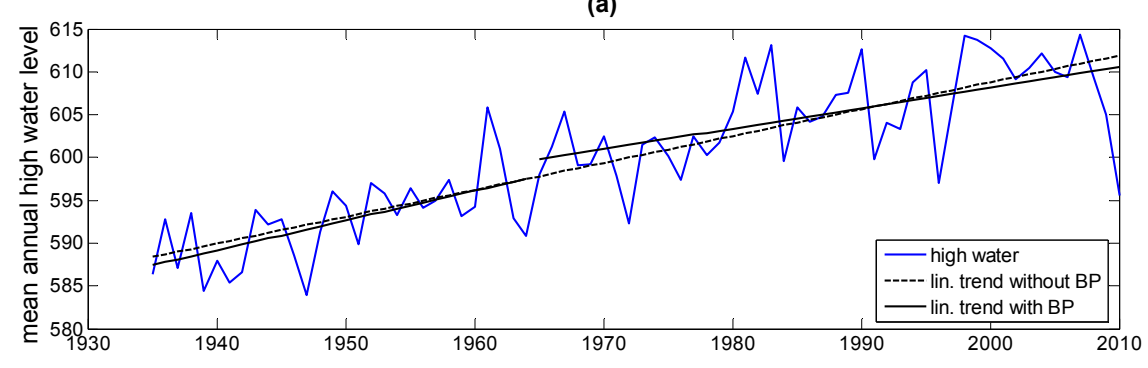

(b)

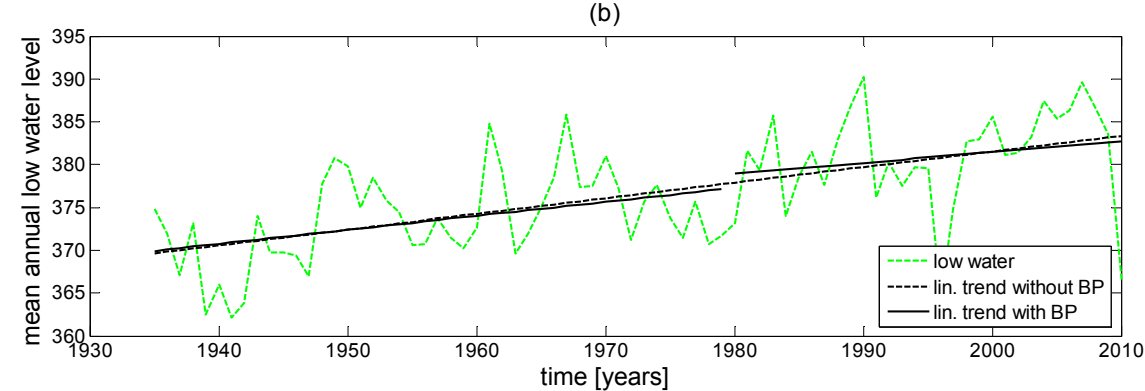

Figure 8. Linear trend annual means high (a) and low water level (b), with and without breakpoint (BP) consideration, gauge Borkum Südstrand, 1935 - 2010

The difference between the continuous and the separated trend is evident. Without consideration of the break in the time series the linear trend might be overestimated. That is important when the results are used for future predictions. The comparison of the trends of both tidal parameters shows also that the rise of the sea levels behaves not in the same way for both parameters. This follows that the possible impact of climate change in tidal region is not only a rise of the MSL, but a more non-linear change of tidal characteristics.

\section{CONCLUSIONS AND OUTLOOK}

By analyzing the past, future developments might be predicted and one can search for options of adaptation to the changing conditions. One very important precondition to state about possible trends in time series or to predict their progress in the future is that these series are homogeneous and consistent. Abrupt changes in the time series can be detected by the breakpoint analysis. Three different methods were presented, two statistical tests from the literature and one developed approach based on fuzzy logic. The results for the gauge Borkum Südstrand as an example for the measurement sites in the German Bight show the importance of this analysis. Different breakpoints were detected. The following plausibility check is always an important step within the data preparation. Afterwards one has to make the decision, whether the breakpoint should be removed from the time series or if it might be kept and considered at the trend analysis. 
The next steps in the current project will be to go on with further investigations on the named tidal parameters. After the breakpoint analysis the most important tides will be filtered out from the time series by a frequency analysis. As the time series are not equidistant the Lomb Periodogramm is used. Finally the trends in the adjusted time series can be investigated because the aim of the research program is the deduction of adaptation strategies for the future.

\section{ACKNOWLEDGMENTS}

This project is part of the research program KLIWAS (Impacts of climatic change on waterways and navigation - Searching for options of adaptation) by the German Federal Ministry of Transport, Building and Urban Development (BMVBS).

\section{REFERENCES}

Alexandersson, H. (1986): A Homogeneity test applied to precipitation data. In: Journal of Climatology, Vol. 6, No. 6, pp. 661-675

Eriksson, B.K., Van der Heide, T., Van de Koppel, J., Piersma, T., Van der Veer, H.W., Olff, H. (2010) Major changes in the ecology of the Wadden Sea: human impacts, ecosystem engineering and sediment dynamics. Ecosystems 13: 752-764.

DIN 4049-3 (1992): Hydrologie. Teil 3: Begriffe zur quantitativen Hydrologie, Berlin: Beuth Verlag $\mathrm{GmbH}$.

Hein, H.; Weiss, R.; Barjenbruch, U.; MAI, S. (2010): Uncertainties of tide gauges \& the estimation of regional sea level rise. Hydro 2010 Rostock - Warnemünde, Conference Proceedings.

Hein, H., Mai, S., Barjenbruch, U. (2011a) What tide gauges reveal about the future sea level, Aqua Alta, Hamburg, Conference Proceedings.

Hein, H., Mai, S., Barjenbruch, U. (2011b) Coastal long term processes, tidal characteristics and climate change, 5th International Short Conference on Applied Coastal Research, Aachen, Conference Proceedings.

Reeves, J.; Chen, J.; Wamg, X. L.; Lund, R.; Lu, Q. Q. (2006): A Review and Comparision of Changepoint Detection Techniques for Climate Data. In: Appl.Meteor.Climatol, Vol. 46, pp. 900915

Sudau, A., Weiss, R (2010): Der Einfluss vertikaler Landbewegungen auf langwellige Wasserstandsänderungen, 40. IWASA - Internationales Wasserbau-Symposium Aachen, 08. Januar 2010.

Wahl, T.; Jensen, J.; Frank, T.; Haigh, I. (24-1-2011): Improved estimates of mean sea level changes in the German Bight over the last 166 years. In: Ocean Dynamics 